\title{
Initiation of Batrachochytrium dendrobatidis infection in the absence of physical contact with infected hosts - a field study in a high altitude lake
}

\author{
Elodie A. Courtois, Adeline Loyau, Mégane Bourgoin and Dirk S. Schmeller
}

E. A. Courtois (courtoiselodie@gmail.com), Laboratoire Ecologie, Evolution, Interactions des Systemes Amazoniens (LEEISA), Univ. de Guyane, CNRS, IFREMER, FR-97300 Cayenne, France, and: Dept of Biology, Univ. of Antwerp, Wilrijk, Belgium. - M. Bourgoin, Station d'écologie expérimentale du CNRS à Moulis, Moulis, France. - A. Loyau and D. S. Schmeller, Helmboltz Centre for Environmental Research - UFZ, Dept of Conservation Biology, Leipzig, Germany. AL and DSS also at: EcoLab, Univ. de Toulouse, CNRS, INPT, UPS, Toulouse, France.

\begin{abstract}
Understanding transmission is a critical prerequisite for predicting disease dynamics and impacts on host populations. It is well established that Batrachochytrium dendrobatidis $(B d)$, the amphibian fungal pathogen responsible for chytridiomycosis, can be transmitted directly, through physical contact with an infected host. However, indirect pathways of transmission remain poorly investigated. We conducted a five-week long field infection experiment at a high altitude mountain lake in the French Pyrenees to investigate $B d$ transmission pathways in larval midwife toads Alytes obstetricans. Uninfected naïve tadpoles were co-housed either with infected tadpoles (direct and indirect transmission) or with uninfected ones (indirect transmission only). We found that physical contact with an infected host is not necessary for initial infection with $B d$ and that all tadpoles became infected after only four weeks. However, physical contact with infected tadpoles led to a faster spread within a tadpole group and resulted in higher $B d$ loads and subsequently higher mortality. Our findings clearly demonstrate that in $A$. obstetricans, $B d$ can quickly spread in a population even without physical contact. Our experiment therefore stresses the importance of indirect transmission of $B d$ zoospores in infected lakes for disease dynamics, especially when a reservoir species such as $A$. obstetricans is present.
\end{abstract}

The fungal pathogen Batrachochytrium dendrobatidis (hereafter $B d$ ) is the aetiological agent of the amphibian disease chytridiomycosis which impacts a broad range of amphibian species and can drive them to extinction (Gascon 2007). Bd is nearly globally distributed and has spread into important amphibian hotspots, such as Madagascar (Bletz et al. 2015) and the Guiana shield (Courtois et al. 2015). While $B d$ can infect a large variety of amphibian species, not all species are heavily impacted by chytridiomycosis. Contrasting outcomes are rapid death in some amphibian species (Lips 1999, Bosch and MartínezSolano 2006, Lips et al. 2006, Rachowicz et al. 2006) and only minor impacts in others (Weldon et al. 2004, Daszak et al. 2005). The variability in outcomes of infection may also be determined by biotic and abiotic factors, such as temperature (Walker et al. 2010), probiotics and innate defence mechanisms (Bletz et al. 2013, Woodhams et al. 2014), pathogen-environment interactions (Schmeller et al. 2014) and species life-history traits (Lips et al. 2003, Bielby et al. 2008).

Transmission is one of the key processes in host-pathogen interactions and understanding it is a critical prerequisite for predicting disease dynamics and impact on a host population (Anderson and May 1991, McCallum et al. 2001).
Pathogens can be transmitted in various ways, including direct physical contact with a host, an intermediate host or a vector. Pathogens can also be transmitted without physical contact, through air, water, soil or other substrates. Indirect transmission is strongly favored by the existence of free-living infective stages, such as the motile, waterborne zoospore of $B d$ (Longcore et al. 1999).

Direct contact between hosts is a major transmission pathway of $B d$. Horizontal transmission has been shown in laboratory and in field enclosures experiments between tadpoles of different amphibian species, and from the larval to the post-metamorphic stage (Rachowicz and Vredenburg 2004, Rachowicz and Briggs 2007, Fernández-Beaskoetxea et al. 2016). Transmission rate is affected by the density of infected individuals (Rachowicz and Briggs 2007), and specific behaviors, such as aggregation, reproductive behaviors (amplexus), and territorial confrontation which can favor Bd transmission (Rowley and Alford 2007). However, in field experiments, it was found that a few individuals became infected in enclosures that did not contain any infected individuals, suggesting that the background density of $B d$ zoospores in the water could be sufficient to trigger infection (Rachowicz and Briggs 2007, FernándezBeaskoetxea et al. 2016). 
In water, infection of an amphibian host by $B d$ zoospores without direct contact with an infected individual can occur through three non-mutually exclusive mechanisms. First, the individual can become infected by swimming in water containing $B d$ zoospores recently released by an infected host. In the laboratory, Nichols et al. (2001) were the first ones to experimentally infect amphibian hosts with $B d$ by dripping a solution containing zoospores onto the back and legs of amphibians. It was then shown that uninfected tadpoles and toadlets can become infected by a simple exposure to water in which $B d$ infected hosts were housed (Carey et al. 2006, Greenspan et al. 2012). These results from laboratory studies clearly demonstrate that physical contact between animals is not always required for $B d$ transmission. In the field, close proximity to infected conspecifics, high density and aggregating behaviors may be important parameters increasing the probability of $B d$ transmission through infected water. Dense aggregation behavior along lake shorelines is known to occur in the tadpoles of several species (Bosch et al. 2001, Rowley and Alford 2007, Venesky et al. 2011). Such behavior most likely benefits tadpoles by promoting thermoregulation (Han et al. 2008), but can also increase the probability that a tadpole becomes infected by multiplying the contacts and increasing the proximity between susceptible and infected tadpoles (Venesky et al. 2011). For example, Venesky et al. (2011) found that the average $B d$ load was significantly higher for Anaxyrus fowleri, a species displaying aggregation behavior, than Hyla versicolor a non-aggregating species.

Second, indirect transmission can depend on the ability of a motile $B d$ zoospore to reach and infect a new host. Laboratory studies using cultured $B d$ showed that zoospores swim an average of $2 \mathrm{~cm}$ on agar plates before encysting (Piotrowski et al. 2004). As agar plates are very different to water in terms of swimming resistance, it is likely that this $2 \mathrm{~cm}$ distance underestimates the swimming capacities of $B d$ zoospores in water. In addition, positive chemotaxis toward constituents of keratin over small distances $(<1 \mathrm{~cm})$ has been reported in $B d$ (Moss et al. 2008, Garmyn et al. 2012). Aggregation, density and proximity of infected individuals are also expected to play an important role when this transmission mechanism is involved.

Finally, the environment itself may bring the pathogen and the host in contact through water and/or substrate movements. Laboratory experiments have shown that $B d$ zoospores can survive in sterile moist creek bed sand at $\mathrm{pH} 5.8$ for more than 12 weeks, suggesting that substrate translocation could favor $B d$ spread (Johnson and Speare 2005). Recently, Hagman and Alford (2015) conducted an elegant study in semi-natural conditions to specifically test the impact of water movements on $B d$ transmission between tadpoles of green-eyed tree frogs Litoria serrata. They deviated stream water to demonstrate that, in a lotic habitat, transmission between tadpoles can occur downstream via waterborne zoospores, even when individuals do not come into physical contact (Hagman and Alford 2015). However, it is not clear yet whether $B d$ transmission and spread can occur without direct contact between hosts in a lentic habitat in which water movements are expected to be lower than in a lotic system.
Indirect transmission of $B d$ is favored by zoospore persistence and infectivity in the environment outside of hosts. In vitro studies have shown that $B d$ zoospores can survive in tap and deionized water and remain infective for three and four weeks respectively (Johnson and Speare 2005). Infectivity has been observed in autoclaved lake water for up to seven weeks (Johnson and Speare 2003). Investigations in natural conditions also detected $B d$ DNA in many different water bodies with a year-round presence, although these results do not necessarily indicate that infective zoospores were present (Walker et al. 2007, Hyman and Collins 2011, Chestnut et al. 2014). Despite shortcomings, these findings suggest that lake water may act as an important source for infective $B d$ zoospores.

The objective of this study was to investigate, in a lentic habitat, whether transmission of $B d$ can occur without physical contact between hosts. The experiment was conducted in the Pyrenees, in a high altitude lake $(2350 \mathrm{~m})$ that has tested positive for $B d$ for several years. We investigated $B d$ transmission pathway in larval Alytes obstetricans, a highly susceptible $B d$ indicator species that has been the focus of several studies across Europe (Bosch et al. 2001, Walker et al. 2010). Alytes obstetricans tadpoles can suffer both lethal and sub-lethal effects of $B d$ infection but generally experience few adverse effects and can remain infected until they reach metamorphosis (Tobler and Schmidt 2010). For this reason, and because they have a long-lived tadpole stage with the ability to overwinter in mountain lakes, they may act as important reservoirs maintaining $B d$ in lake water (Fernández-Beaskoetxea et al. 2016), even in the absence of post-metamorphic hosts (Rachowicz and Vredenburg 2004, Bielby et al. 2008, Briggs et al. 2010).

Our experiment consisted of two treatments that differed in the presence or absence of infected individuals in groups of uninfected naïve tadpoles. In the first treatment, infected and uninfected $A$. obstetricans tadpoles were placed in close contact, which allowed for both direct and indirect transmission of $B d$. In contrast, in the second treatment, direct physical contact between infected and uninfected tadpoles was prohibited, therefore, at the onset of the experiment, only indirect transmission could occur in this treatment. Our objectives were 1) to determine whether the background source of $B d$ zoospores in the lake water was sufficient to trigger infection, and 2) to examine the trajectories of infections in both treatments.

\section{Methods}

\section{Experiment}

We conducted this field study at Lake Madamete (HautesPyrénées, France, $\left.42^{\circ} 51^{\prime} 48.1^{\prime \prime} \mathrm{N}, 0^{\circ} 08^{\prime} 35.8^{\prime \prime} \mathrm{E}\right)$. This lake is situated in the Parc National des Pyrénées at an elevation of $2350 \mathrm{~m}$ and has an area of $3500 \mathrm{~m}^{2}$. The mean daily surface water temperature of Lake Madamete ranged between $16.0^{\circ} \mathrm{C}$ and $20.2^{\circ} \mathrm{C}$ (average surface temperature across the experimental period $=18.0^{\circ} \mathrm{C}, \mathrm{SD}=1.2^{\circ} \mathrm{C}$ ) during the course of our experiment (Supplementary material Appendix 1 Fig. A1). This temperature range falls within the optimal growth temperature spectrum of $B d$ of $17-23^{\circ} \mathrm{C}$ (Piotrowski 
et al. 2004). The $\mathrm{pH}$ value was found to be 8.2 in the year of the experiment, which may lead to a slower growth of $B d$ (Piotrowski et al. 2004). Lake Madamete hosted two amphibian species, Rana temporaria (common frog) and Alytes obstetricans (midwife toad) at the time the experiment was conducted. While $R$. temporaria exhibits low susceptibility to $B d, A$. obstetricans is a highly susceptible species (Tinsley et al. 2015). Infection of $A$. obstetricans in the lake was first detected in 2006 with a prevalence of $10 \%$. Since 2011, prevalence of $B d$ has reached near 100\% (Schmeller et al. 2014) and has coincided with a marked decline of this species through mass mortality events of metamorphs.

For this experiment, we collected 60 uninfected tadpoles of $A$. obstetricans from two troughs situated $23 \mathrm{~km}$ away from Lake Madamete (Hautes-Pyrénées, France, 4259'53.4" N, $0^{\circ} 03^{\prime} 19.2^{\prime \prime} \mathrm{E}$, elevation $1000 \mathrm{~m}$, and $42^{\circ} 59^{\prime} 11.2^{\prime \prime} \mathrm{N}$, $0^{\circ} 03^{\prime} 08.5^{\prime \prime} \mathrm{E}$, elevation $1050 \mathrm{~m}$ ). Both trough populations have tested negative for $B d$ since the start of their surveillance in 2009 and therefore very likely have no history of exposure to $B d$. Naïve populations are usually most at risk from the most severe consequences of infection (Anderson and May 1991) and may therefore be a priority for further investigation (Bielby et al. 2013). Uninfected tadpoles were caught on 9 July 2012 at Gosner stages 26 to 41. In addition, the next day, we also collected 30 infected tadpoles of A. obtetricans from the experimental site (Lake Madamete). These tadpoles were caught at Gosner stages between 38 and 40 .

All individuals (trough tadpoles as well as Madamete tadpoles) were weighed and swabbed to assess their infection status before set up of the experiment. Animals were also individually marked using visible implant alpha tags before the beginning of the experiment. For the marking, tadpoles were immobilized by immersion in ice-cold water $\left(\max .4^{\circ} \mathrm{C}\right)$ and restrained by gently squeezing the body between the thumb and index finger during injection. VI alpha tags $(1.2 \times 2.7 \mathrm{~mm}$ tags $)$ were inserted subcutaneously on the ventral side of the tadpoles. After tagging, the unique code remained visible to the naked eye under day light with the option of enhanced visibility under UV-light if necessary. This method of marking has been tested and shown to have no influence on the mortality rate of tadpoles (Courtois et al. 2013).

Our experiment included two treatments. In treatment NI (non-infected), six uninfected tadpoles from the trough populations were housed together in bow nets (diameter $=0.5$ $\mathrm{m}$, height $=0.5 \mathrm{~m}$, Fig. $1 \mathrm{~A}$ ) which were tethered in Lake Madamete; this treatment was replicated five times, exposing
(A)

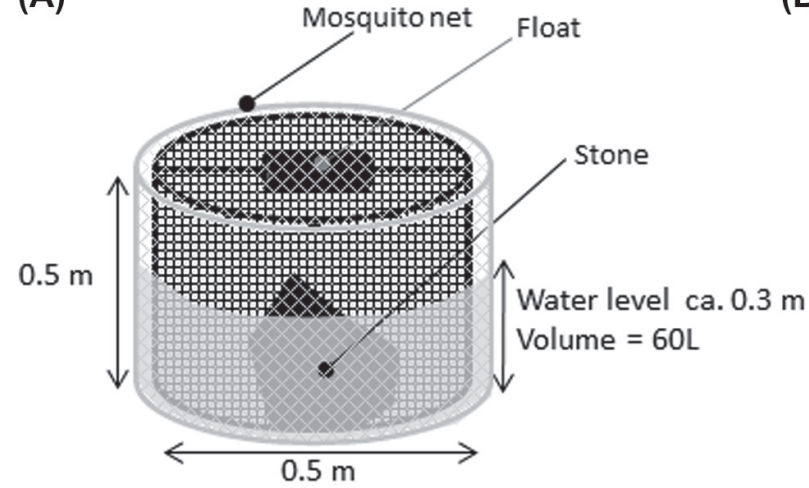

(C)
(B)

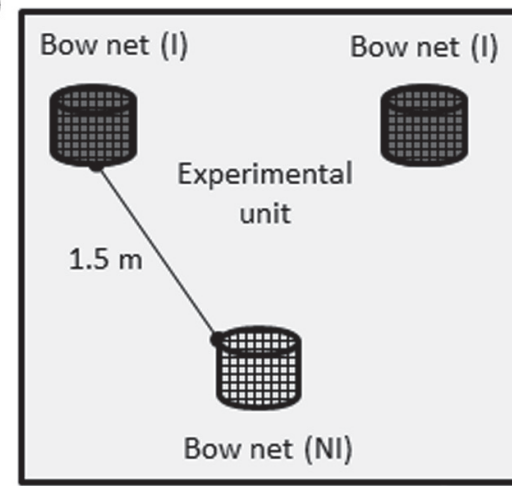

용

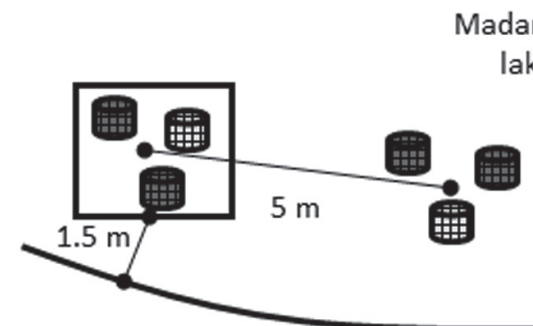

Madamete

lake
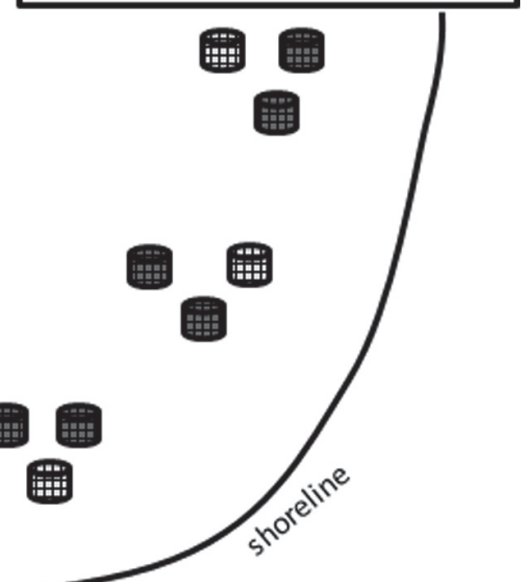

Figure 1. Experimental setup. (A) Single bow net: a float was installed to maintain the net above water level to allow breathing of tadpoles and metamorphs. The stone at the bottom of each bow net allowed metamorphs to climb on and to maintain the net in place. The whole bow net was surrounded by a mosquito net in a distance of minimum three centimeters to prevent contact between tadpoles occurring in the lake and in our bow nets. (B) Experimental unit: each consisted of one bow net of treatment NI (six uninfected tadpoles from Bd naïve populations co-housed in the bow net, light nets) plus two nets of treatment I (three uninfected tadpoles from $B d$ naïve populations co-housed with three infected tadpoles from Lake Madamete in the bow net, dark nets). (C) Schematic of the overall experimental setup with five experimental units along the shoreline of Lake Madamete with indications of distances between the different units and from the shoreline. 
a total of 30 uninfected individuals to zoospores that might be carried by lake water. For treatment I (infected), three infected tadpoles from Lake Madamete were cohoused with three uninfected tadpoles from the trough populations in a bow net, replicated 10 times (Fig. 1A), exposing a total of 30 uninfected individuals to both infected tadpoles and zoospores that might be carried by lake water. The 30 infected tadpoles originating from Lake Madamete were only a means to simulate physical contact with infected tadpoles in treatment I, while having the same tadpole density in both treatments (i.e. six tadpoles per bow net). Usually, A. obstetricans tadpoles display aggregation behavior generating groups of hundreds of individuals. Therefore the density in our experiment may be considered as at the lower end of natural densities.

Each bow net was fixed so that a part was maintained above the water surface to allow the tadpoles and metamorphosing toadlets to breathe (Fig. 1A). Each bow net was covered with a mosquito net so that tadpoles naturally occurring in the lake could not approach the I and NI replicates closer than three centimeters and this distance was similar between the two treatments. Therefore, there was no physical contact between the lake tadpoles and the experimental tadpoles. The chosen distance of three centimeters was selected based on it being slightly larger than the previously reported $2 \mathrm{~cm}$ maximum swimming distance of $B d$ zoospores measured under laboratory conditions (Piotrowski et al. 2004). Lake Madamete, like many high altitude lakes, has very few periphyton species, and we did not observe a periphyton layer building up on our bow nets and mosquito nets over the course of the project, which could have influenced the outcome of our experiment. We detected very few $R$. temporaria or $A$. obstetricans tadpoles $(\mathrm{n}<4)$ in $2 \mathrm{~m}$ distance to our experimental set up on each visit.

The I and NI bow nets were replicated in five experimental units (A to E), each of them containing one NI plus two I bow nets (Fig. 1B), with the positions of the I and NI bow net varying with respect to one another and to the shoreline (Fig. 1C). Distances between bow nets within an experimental unit were $\sim 1.5 \mathrm{~m}$ and distances between the experimental units were $\sim 5 \mathrm{~m}$, with experimental units being at least $1.5 \mathrm{~m}$ from the lake border. Setting up experiment units allowed that I and NI tadpoles experienced similar environmental conditions within a given experimental unit, e.g. in terms of temperature, light, food availability, water movement or proximity to lake resident amphibians. The only drawback to this setting is that tadpoles of groups I could serve as a source of zoospores to the tadpoles of groups $\mathrm{NI}$, however the $\sim 1.5 \mathrm{~m}$ distance is much larger than the swimming distance described for $B d$ zoospores (Piotrowski et al. 2004). The experiment was conducted in a section of the lake with no influence from inflows or outflows of water, to reduce water movements to surface water movements driven by wind.

The naive tadpoles of the two treatments were randomly distributed amongst the different bow nets in regard to their Gosner stage. However, the initial mean mass measured in grams $(\mathrm{g})$ of all uninfected tadpoles in treatment I was higher than in treatment NI (mass ${ }_{\mathrm{NI}} 1.159 \pm 0.0 .276$; mass $_{\mathrm{I}}=1.675 \pm 0.761$; Mann-Whitney U-test: $Z_{59}=3.947$; $\mathrm{p}<0.001$ ). We therefore corrected for a potential confounding effect of uninfected tadpole mass on the outcome of our experiment (see below and supplementary material). The tadpoles collected from Lake Madamete were distributed randomly in regard to their mass to the different bow nets of treatment I (experimental unit A: $5.912 \mathrm{~g} \pm 0.256$; B: $5.833 \mathrm{~g} \pm 0.626$; C: $5.263 \mathrm{~g} \pm 0.408, \mathrm{D}: 5.813 \mathrm{~g} \pm 0.293$; E: $4.993 \mathrm{~g} \pm 0.495)$.

The experimental site was visited for five consecutive weeks, usually at the same weekday $(9,17,24$ and 31 July 2012 and 6 and 13 August 2012). During these visits, the tadpoles were swabbed and weighed to the nearest $0.01 \mathrm{~g}$. Dead tadpoles and metamorphs were removed from the bow nets and stored in 70\% ethanol and surviving metamorphs were brought back to our animal facility. The experiment ended when most of $A$. obstetricans tadpoles in the lake had gone through metamorphosis. On the last visit (13 August), tadpoles originating from Lake Madamete were released and all other tadpoles were brought back to the lab and euthanized.

\section{Bd analysis}

$B d$ DNA was extracted from swabs using a PrepMan extraction method (Hyatt et al. 2007). Briefly, $60 \mu \mathrm{l}$ of PrepMan Ultra was added to each sample (tip of the swab) along with 30 to $40 \mathrm{mg}$ of silica beads $(0.5 \mathrm{~mm}$ diameter) in a $1.5 \mathrm{ml}$ Eppendorf tube. The sample was then homogenized for $45 \mathrm{~s}$ in a Mini Beadbeater 16 and centrifuged for $30 \mathrm{~s}$ at $14000 \mathrm{rpm}$. These steps were repeated twice and tubes were then heated at $96^{\circ} \mathrm{C}$ for $10 \mathrm{~min}$, cooled for $2 \mathrm{~min}$ and centrifuged at $14000 \mathrm{rpm}$ for $3 \mathrm{~min}$. Of the supernatant 20-40 $\mu \mathrm{l}$ was then pipetted and used to test for $B d$ presence. Presence of $B d$ DNA was assessed with quantitative real-time PCR (Hyatt et al. 2007). Each plate included an internal positive control (Internal Positive Control Reagents) to signal amplification inhibition. Each 96-well assay plate also included standards of known $B d$ quantity (control samples containing DNA from 100, 10, 1 and $0.1 B d$ genome equivalents - GE) and controls with no DNA template. These standards were used to quantify $B d$ load for each sample (Boyle et al. 2004, Hyatt et al. 2007). As our highest positive control standard was $100 \mathrm{GE}$, all $B d$ load values above $100 \mathrm{GE}$ might include uncertainty. To prevent inhibition by the extraction reagent, the extractions were diluted by a factor of 10 with double distilled water prior to PCR analysis. A sample was defined as $B d$-positive when the $B d$ load was greater than $0.1 \mathrm{GE}$, a threshold commonly used in other studies (Tobler et al. 2012).

\section{Statistical analysis}

We used generalized linear mixed models (GLMMs) with SAS to analyze our data. We first built a model to examine the changes in prevalence in the two treatments across time. Prevalence, the dependent variable, had a binomial distribution of error terms (infected: 1, uninfected: 0 ), with a logit link function. Day of swabbing, treatment (I, NI) and their interaction were entered as fixed factors in the model. We further included the bow net nested within the experimental unit as well as the tadpole identity nested within the bow net as random factors in our model. 
We investigated the parameters explaining the individual $B d$ load in each treatment in the following ways. Given that the tadpole mass was not the same in the two treatments, we first compared the slopes of the relationship between logtransformed $B d$ load (log GE) and log-transformed tadpole body mass (log mass). As the slopes did not differ between treatments $\left(t_{329}=-0.051 ; \mathrm{p}=0.959\right.$; Supplementary material Appendix 1 Fig. A2), we proceeded by log-transforming both $B d$ load expressed as genome equivalents (GE) and the tadpole mass. We then built a GLMM. The dependent variable of the model was log GE (with a normal distribution of error terms). The independent, explanatory variables entered in the model as fixed effects were day, treatment (I, $\mathrm{NI}$ ) and their interaction. To take into account the difference in initial tadpole mass between the two treatments we also included log mass as a covariate (fixed effect) in the model. As in the previous GLMM, we also included the bow net nested within the experimental unit as well as the tadpole identity nested within the bow net as random factors in our model.

$B d$ infection dynamics could be driven by the density of infected individuals as well as the availability of $B d$ zoospores in a bow net. To explore these possibilities, we conducted a rank correlation analysis. We first calculated the density of all newly and initially infected tadpoles (minus mortalities) for each sampling day ( $\mathrm{N}$ infected individuals $\times$ liter $^{-1}$ ) and the estimated density of $B d$ zoospores (sum of GE across all infected individuals including those from Lake Madamete per bow net $\times\left(\right.$ liter $\left.^{-1}\right)$. Then, we correlated these two variables with the number of infected individuals, observed one week and two weeks later in the same bow net.

Mortality was compared between the two treatments using a $\chi^{2}$-test. As mortalities occurred in the two last weeks of the experiment only (four mortalities in week 4 and four mortalities in week 5) and always at metamorphosis (tadpoles at Gosner stage 45 or 46) there were insufficient data to run a survival analysis using the Kaplan-Meier method (Kaplan and Meier 1958). In treatment I 19/30 tadpoles reached metamorphosis compared to $20 / 30$ tadpoles in treatment NI.

\section{Data deposition}

Data available from the Dryad Digital Repository: <http:// dx.doi.org/10.5061/dryad.20h6m > (Courtois et al. 2016).

\section{Results}

$B d$ prevalence in originally uninfected tadpoles increased during the course of the experiment and reached $100 \%$ in all experimental units at the end. Prevalence dynamics in all bow nets of treatment NI were similar to each other with low prevalence at day 0,8 and 15 of the experiment and then a very rapid increase to $100 \%$ at day 22 (Supplementary material Appendix 1 Fig. A3). The prevalence increased more rapidly in treatment $\mathrm{I}$, although this difference in the infection dynamics was not significant (Day: $\mathrm{F}_{1,297}=94.08$; $\mathrm{p}<0.001$; Treatment: $\mathrm{F}_{1,297}=1.42 ; \mathrm{p}=0.234$; Day $\times$ Treatment: $F_{1,297}=0.77 ; p=0.382$; Fig. 2A). Prevalence of tadpoles from Madamete used in treatment I remained at $100 \%$ during the course of the experiment.

$B d$ load developed significantly differently in the two treatments with a faster increase of the infection load in treatment I compared to treatment NI (Day: $\mathrm{F}_{1,322}=309.67$; $\mathrm{p}<0.001$; Treatment: $\mathrm{F}_{1,41.7}=1.29 ; \mathrm{p}=0.2630 ;$ Day $\times$ Treatment: $\mathrm{F}_{1,283.2}=10.29 ; \mathrm{p}=0.0015$; tadpole mass: $\mathrm{F}_{1,417.4}=18.50 ; \mathrm{p}<0.001$; Fig. $2 \mathrm{~B}$ ). The dynamics per bow net within both treatments were similar (Supplementary material Appendix 1 Fig. A4). In bow nets of treatment I, we observed the biggest increase of $B d$ load between day 15 and day 22, while in bow nets of treatment NI, the biggest increase of $B d$ load was observed between day 28 and day 35 (Fig. 2B, Supplementary material Appendix 1 Fig. A4). In treatment $\mathrm{I}$, we observed a maximum individual infection load of $50370 \mathrm{Bd}$-GE, as compared to $15900 \mathrm{Bd}$-GE in treatment NI.

The density of infected tadpoles in a bow net (= newly infected tadpoles + initially infected tadpoles) did not predict the number of infected naïve individuals observed one week later $\left(t_{+7}: R_{73}=-0.184 ; p=0.163\right)$. However, the density of infected individuals predicted well the number of infected individuals two weeks later $\left(\mathrm{t}_{+14}=\mathrm{R}_{58}=0.509\right.$; $\mathrm{p}<0.001)$. The estimated density of $B d$ zoospores was not a good predictor of the number of infected individuals observed one or two weeks later $\left(t_{+7}: R_{73}=-0.030 ; t_{+14}\right.$ : $\mathrm{R}_{58}=-0.206 ; \mathrm{p}>0.142$ ).

In total we observed eight mortalities among previously uninfected experimental tadpoles ( $13 \%$ of all), all occurring in the last two weeks of the experiment, with a significant difference between the two treatments (7/30 mortalities in the treatment $\mathrm{I}$ versus $1 / 30$ in the treatment NI; $\chi_{1}^{2}=4.039, p=0.044$, Fig. 3). Mortality was even higher in tadpoles coming from Lake Madamete used in treatment I, with 14/30 (46.6\%) mortalities. All mortalities occurred at metamorphosis at Gosner stage 45 or 46.

\section{Discussion}

A fundamental component to understand disease dynamics at the species and population level is determining the mechanisms of transmission under natural conditions. Our field experiment conducted in a lentic habitat confirmed that $B d$ infections in high altitude lakes can be initiated in naïve individuals in the absence of physical contact with infected hosts. Surprisingly, infections within groups of uninfected individuals were initiated almost as rapidly as within groups containing infected tadpoles and after 35 days there were no substantial differences in $B d$ prevalence. Our results indicate that the lake contained sufficient sources of infectious $B d$ zoospores to trigger $B d$ infections among naïve individuals. Our experiment therefore delivers additional evidence that $B d$ is highly transmissible in mountain lakes, explaining the high prevalence in mountainous habitats reported earlier (Walker et al. 2010). In our experiment we also observed that once infection was established in a group of previously naïve tadpoles, the propagation was rapid within a group, with all tadpoles being $B d$ infected after only four weeks. Nevertheless, tadpoles in treatment NI had an infection burden that was about three times lower than the burden of 

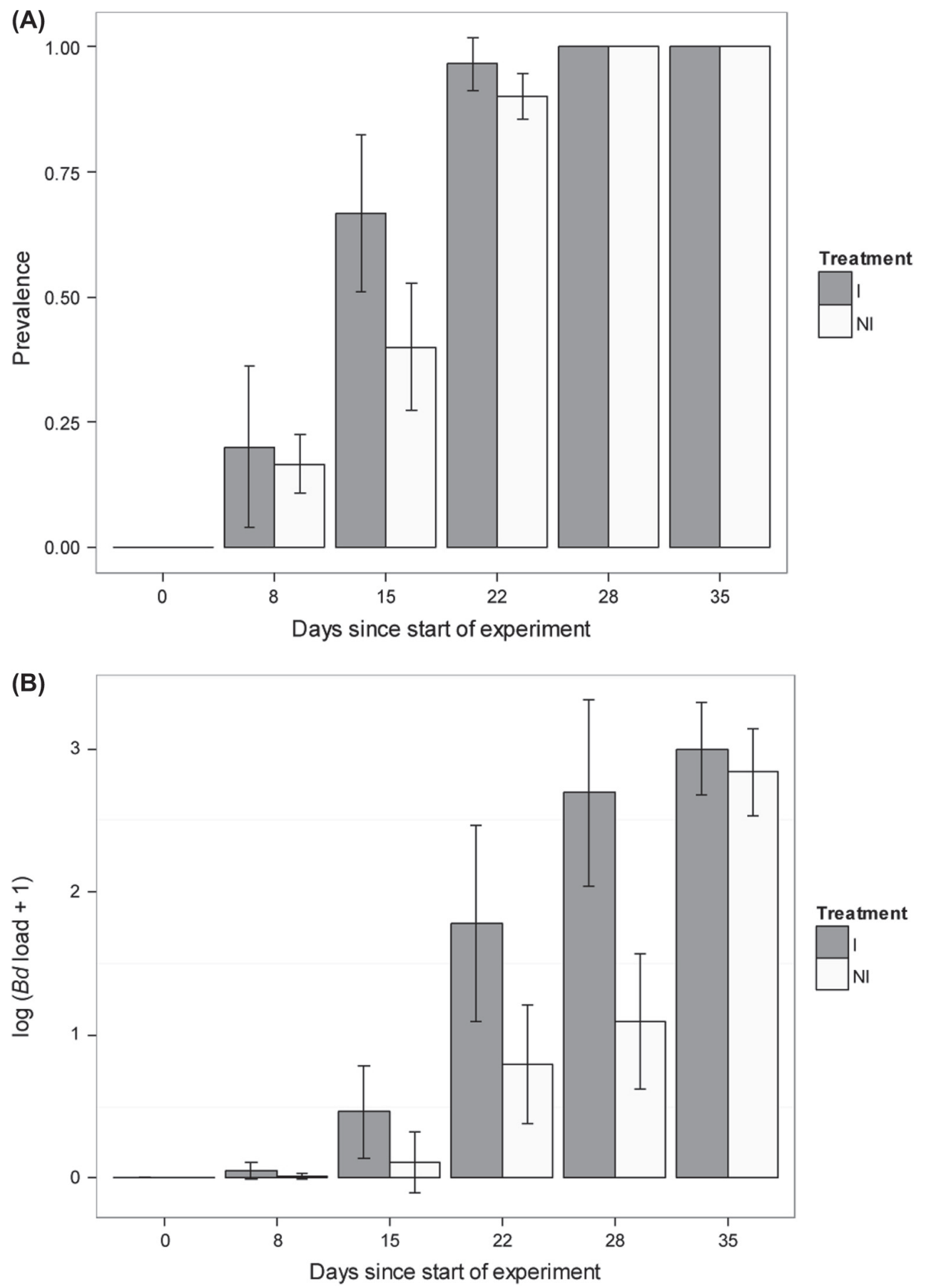

Figure 2. The trajectory of (A) $B d$ prevalence and (B) $\log (B d \operatorname{load}+1)$ in the two treatments across experimental individuals from the $B d$ naïve populations averaged for all bow nets. Error bars represent standard deviation around the mean value. NI $=$ treatment with only uninfected tadpoles from $B d$ naïve populations, $\mathrm{I}=$ treatment with three uninfected tadpoles ( $B d$ naïve populations) cohoused with three infected tadpoles (from Lake Madamete).

individuals in treatment I, and this translated in comparably lower mortality.

Earlier experiments on the same (Fernández-Beaskoetxea et al. 2016) and other (Rachowicz and Briggs 2007) amphibian species found that some tadpoles could become infected by $B d$ without direct contact with infected hosts. Our study generalizes this fact and shows a rapid spread of the pathogen within tadpole cohorts can be reached even with low tadpole densities. Once $B d$ infection was initiated in a group of tadpoles, the infection spread rapidly in the group after ca two weeks. This time period likely corresponds to the growth dynamics of $B d$, as the environmental conditions in Lake Madamete were near ideal growth conditions for $B d$. Under such conditions $B d$ may reach a growth plateau after $12-15$ days (Piotrowski et al. 2004, Voyles et al. 2012). In our experiment, we observed 


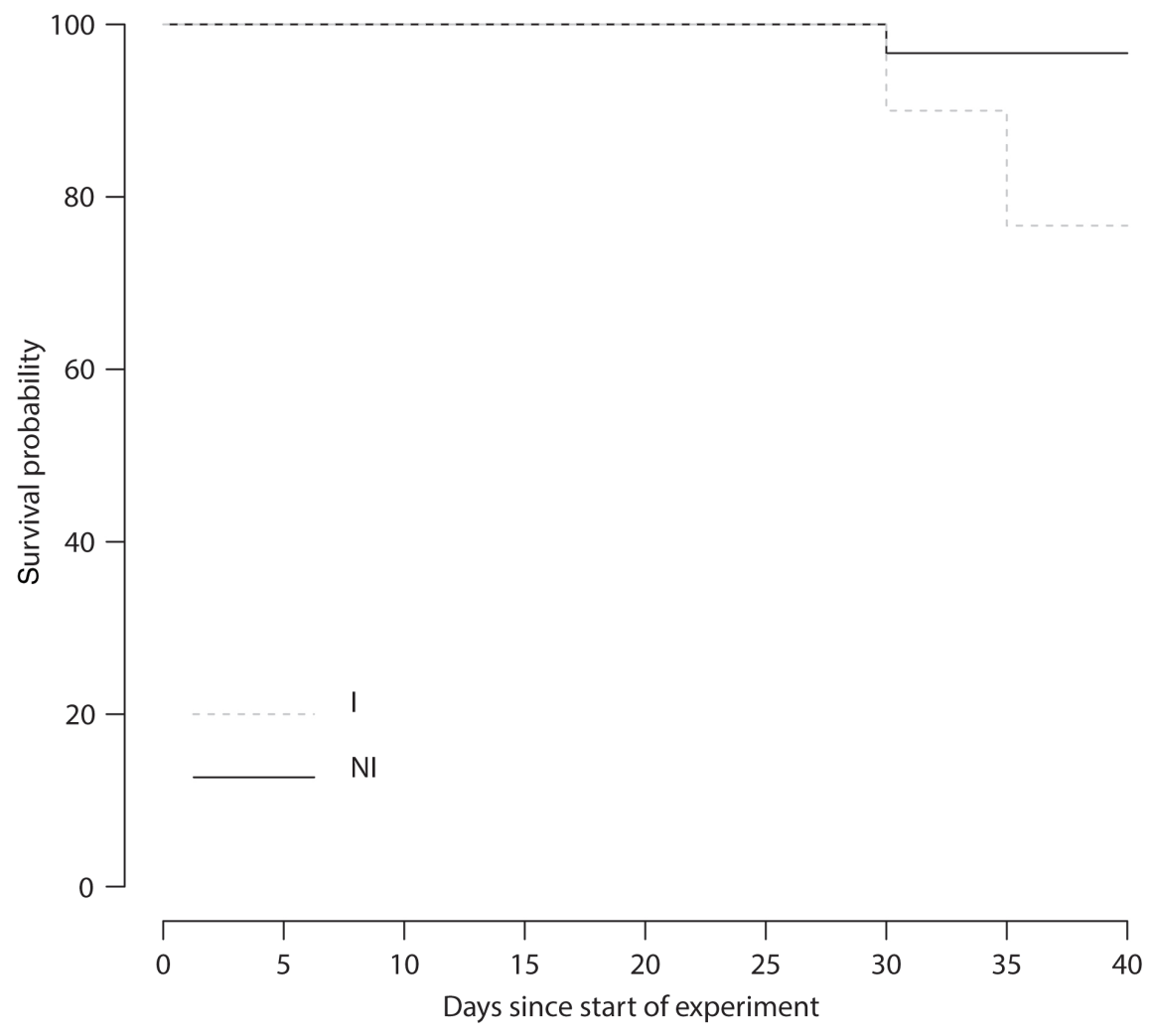

Figure 3. Survival of tadpoles by treatment. NI = treatment with only uninfected tadpoles ( $B d$ naïve populations), $I=$ treatment with three uninfected tadpoles ( $B d$ naïve populations) cohoused with three infected tadpoles (from Lake Madamete).

an increased infection pressure after two weeks, as likely more $B d$ zoospores were released from newly infected tadpoles corresponding to the plateau phase of $B d$ growth cycle. Hence, in a system with $A$. obstetricans tadpoles present, these early infected tadpoles likely act as multipliers of infectious $B d$ zoospores (Fernández-Beaskoetxea et al. 2016). Within each bow net the contact with infected tadpoles then determined the infection dynamics after the initial infection, being also dependent on the density of infected tadpoles (Rachowicz and Briggs 2007). This relationship then explains the difference of infection dynamics in both treatments at the beginning and the lack of it at the end of the experiment.

Difference in mortalities between the two treatments is likely explained by the difference in the initial infection pressure translating to different infection loads. Tadpoles co-housed with infected tadpoles from Lake Madamete experienced a higher infection pressure, had higher $B d \mathrm{GE}$ values, and a higher mortality rate at the end of the experiment compared to tadpoles that were initially uninfected. Alytes obstetricans tadpoles from Lake Madamete were likely an important source of $B d$ zoospores, having been infected since a longer time, acting as $B d$ reservoirs (FernándezBeaskoetxea et al. 2016). Indeed, two mathematical models showed that a larger initial $B d$ zoospore pool is expected to led to a stronger impact of $B d$ on host populations (Briggs et al. 2010, Woodhams et al. 2011). Our semi-natural experiment is in line with earlier studies on aggregation behavior (Rowley and Alford 2007, Venesky et al. 2011). In aggregating species, and hence also in A. obstetricans, infection can spread more rapidly due to an increased infection probability through more frequent contacts between infected and uninfected individuals as compared to nonaggregating amphibian species.

Infectious $B d$ zoospores responsible for the initiation of infection within groups of previously uninfected tadpoles may come from various sources; however, our experimental study was not specifically designed to discriminate between these sources and our results only allow speculating about the mechanisms underlying indirect transmission. Amphibian hosts were the most probable source of infectious $B d$ zoospores. The necessity to offer comparable local environmental conditions to both experimental treatments led us to install the initially uninfected tadpoles at a $1.5 \mathrm{~m}$ distance from the groups containing infected tadpoles. In addition, Lake Madamete also contained tadpoles of Rana temporaria and A. obstetricans tadpoles in its shallowest zones. While we installed our experimental units at least $30 \mathrm{~m}$ away from these zones, we occasionally observed a tadpole approaching our bow nets. In most cases, these tadpoles were of $R$. temporaria, which have proven to have low susceptibility to $B d$ in Lake Madamete ( $<5 \%$; Schmeller unpubl.). Although we cannot rule out that some of the tadpoles may have been infected and released $B d$ zoospores in the proximity of the bow nets, we consider any impact this may have had on our experiment to be marginal. In contrast, the Madamete tadpoles housed in experimental bow nets of the treatment I represent a more likely source of infectious zoospores, due to their high infection burden and their proximity to NI tadpoles $(\sim 1.5 \mathrm{~m})$. In any case, our experiment suggests that the dispersal distance 
of $B d$ zoospores might be greater than the two centimeters observed in an earlier study (Piotrowski et al. 2004).

Lake water and/or substrate is an alternative, yet less probable, source of $B d$ zoospores. Indeed, studies in the laboratory have shown that $B d$ zoospores have a relatively high longevity and maintained infectivity in the absence of a host in sterile lake water and moist soil (Johnson and Speare 2003, 2005). There is also evidence that the pathogen may survive saprophytically and even produce zoosporangia and zoospores in the absence of an amphibian host (Johnson and Speare 2003, Garmyn et al. 2012). However, how long infective $B d$ zoospores are able to persist in nature still remains to be investigated.

Dispersal of $B d$ zoospores from environmental source and/or infected hosts to naïve experimental tadpoles may have occurred through several mechanisms. $B d$ zoospores are motile and able to swim in order to reach a new host. It has also been demonstrated that they show chemotaxis towards keratinized skin at least over a $1 \mathrm{~cm}$ distance (Moss et al. 2008, Garmyn et al. 2012). In addition, while we conducted the experiment in the part of the lake where water movements are reduced, they still may have contributed to carry zoospores from infected hosts to uninfected individuals, as already shown in a lotic water system (Hagman and Alford 2015).

Our study conducted in a lentic freshwater system stresses the importance of sources of $B d$ zoospores and their effectiveness in initiating $B d$ infection even in the absence of physical contact with infected hosts in a mountain lake ecosystem. This is of even more importance as species like $A$. obstetricans, $R$. sierra and $R$. mucosa have long-lived tadpoles which reach high $B d$ loads over time and serve as $B d$ reservoirs in lake water. Alytes obstetricans is now also a confirmed vector for $B d$ to other species (FernándezBeaskoetxea et al. 2016), and is likely inflicting long lasting effects on larval survival and therewith recruitment rates in conspecifics as well as other amphibian hosts. Our experiment also stresses that one full cycle of $B d$ growth may be enough to quickly reach uninfected hosts and spread through cohorts of conspecifics. Therefore, future mitigation strategies of chytridiomycosis should aim at reducing/ controlling sources of zoospores at the habitat level. The latter might be achieved by using natural augmentation of zooplankton species by removal of introduced fish and release of laboratory bred indigenous zooplankton species (Schmeller et al. 2014).

Acknowledgements - We thank the Parc National des Pyrénées (PNP) and especially Linda Rieu, Eric Sourp and Dominique Oulieu for providing authorizations and facilities for this experiment. We thank the Direction de l'Environnement de l'Aménagement et du Logement (DREAL) of Midi-Pyrénées, Laurie Esparza for help with the administrative procedure (Permit number: 2009-11), Marjorie Puertolas and Gilles Pottier for help in the field and Kieran Bates for improving the English. We also thank Hamish McCallum for comments on earlier versions of this manuscript.

Funding - This work was supported by a grant from the BiodivERsA project RACE (Risk Assessment of Chytridiomycosis to European amphibian biodiversity; Fisher et al. 2012; <www. bd-maps.eu $>$ ). AL is currently funded by a Marie SkłodowskaCurie fellowship.

\section{References}

Anderson, R. and May, R. 1991. Infectious disease of humans. Dynamics and control. - Oxford Univ. Press.

Bielby, J. et al. 2008. Predicting susceptibility to future declines in the world's frogs. - Conserv. Lett. 1: 82-90.

Bielby, J. et al. 2013. Geographic and taxonomic variation in Batrachochytrium dendrobatidis infection and transmission within a highly endemic amphibian community. - Divers. Distrib. 19: 1153-1163.

Bletz, M. C. et al. 2013. Mitigating amphibian chytridiomycosis with bioaugmentation: characteristics of effective probiotics and strategies for their selection and use. - Ecol. Lett. 16: 807-820.

Bletz, M. C. et al. 2015. Widespread presence of the pathogenic fungus Batrachochytrium dendrobatidis in wild amphibian communities in Madagascar. - Sci. Rep. 5:8633.

Bosch, J. and Martínez-Solano, I. 2006. Chytrid fungus infection related to unusual mortalities of Salamandra salamandra and Bufo bufo in the Peñalara Natural Park, Spain. - Oryx 40: 84-89.

Bosch, J. et al. 2001. Evidence of a chytrid fungus infection involved in the decline of the common midwife toad (Alytes obstetricans) in protected areas of central Spain. - Biol. Conserv. 97: 331-337.

Boyle, D. et al. 2004. Rapid quantitative detection of chytridiomycosis (Batrachochytrium dendrobatidis) in amphibian samples using real-time Taqman PCR assay. - Dis. Aquat. Organisms 60: $141-148$.

Briggs, C. J. et al. 2010. Enzootic and epizootic dynamics of the chytrid fungal pathogen of amphibians. - Proc. Natl Acad. Sci. USA 107: 9695-9700.

Carey, C. et al. 2006. Experimental exposures of boreal toads (Bufo boreas) to a pathogenic chytrid fungus (Batrachochytrium dendrobatidis). - EcoHealth 3: 5-21.

Chestnut, T. et al. 2014. Heterogeneous occupancy and density estimates of the pathogenic fungus Batrachochytrium dendrobatidis in waters of North America. - PloS ONE 9: e106790.

Courtois, E. A. et al. 2013. The use of visible implant alpha tags for Anuran tadpoles. - Herpetol. Rev. 44: 231-233.

Courtois, E. A. et al. 2015. Widespread occurrence of $B d$ in French Guiana, South America. - PloS ONE 10(4): e0125128.

Courtois, E. A. et al. 2016. Data from: Initiation of Batrachochytrium dendrobatidis infection in the absence of physical contact with infected hosts - a field study in a high altitude lake. - Dryad Digital Repository, <http://dx.doi.org/10.5061/ dryad. $20 \mathrm{~h} 6 \mathrm{~m}>$.

Daszak, P. et al. 2005. Amphibian population declines at Savannah river site are linked to climate, not chytridiomycosis. - Ecology 86: 3232-3237.

Fernández-Beaskoetxea, S. et al. 2016. Infection and transmission heterogeneity of a multi-host pathogen (Batrachochytrium dendrobatidis) within an amphibian community. - Dis. Aquat. Organisms 118: 11-20.

Fisher, M.C. et al. 2012. RACE: risk assessment of chytridiomycosis to European amphibian biodiversity. - Froglog 101: 45-47.

Garmyn, A. et al. 2012. Waterfowl: potential environmental reservoirs of the chytrid fungus Batrachochytrium dendrobatidis. - PloS ONE 7: e35038.

Gascon, C. 2007. Amphibian conservation action plan: proceedings IUCN/SSC Amphibian Conservation Summit 2005. - IUCN.

Greenspan, S. E. et al. 2012. Transmission of Batrachochytrium dendrobatidis to wood frogs (Lithobates sylvaticus) via a bullfrog (L. catesbeianus) vector. - J. Wildl. Dis. 48: 575-582. 
Hagman, M. and Alford, R. A. 2015. Patterns of Batrachochytrium dendrobatidis transmission between tadpoles in a high-elevation rainforest stream in tropical Australia. - Dis. Aquat. Organisms 115: 213-221.

Han, B. A. et al. 2008. Ancient behaviors of larval amphibians in response to an emerging fungal pathogen, Batrachochytrium dendrobatidis. - Behav. Ecol. Sociobiol. 63: 241-250.

Hyatt, A. et al. 2007. Diagnostic assays and sampling protocols for the detection of Batrachochytrium dendrobatidis. - Dis. Aquat. Organisms 73: 175-92.

Hyman, O. J. and Collins, J. P. 2011. Evaluation of a filtrationbased method for detecting Batrachochytrium dendrobatidis in natural bodies of water. - Dis. Aquat. Organisms 97: $185-195$.

Johnson, M. L. and Speare, R. 2003. Survival of Batrachochytrium dendrobatidis in water: quarantine and disease control implications. - Emerging Infectious Dis. 9: 922-925.

Johnson, M. L. and Speare, R. 2005. Possible modes of dissemination of the amphibian chytrid Batrachochytrium dendrobatidis in the environment. - Dis. Aquat. Organisms 65: 181-186.

Kaplan, E. L. and Meier, P. 1958. Nonparametric estimation from incomplete observations. - J. Am. Stat. Ass. 53: 457-481.

Lips, K. R. 1999. Mass mortality and population declines of anurans at an upland site in western Panama. - Conserv. Biol. 13: 117-125.

Lips, K. R. et al. 2003. Ecological traits predicting amphibian population declines in Central America. - Conserv. Biol. 17: 1078-1088.

Lips, K. R. et al. 2006. Emerging infectious disease and the loss of biodiversity in a Neotropical amphibian community. - Proc. Natl Acade. Sci. USA 103: 3165-3170.

Longcore, J. E. et al. 1999. Batrachochytrium dendrobatidis gen. et sp. nov., a chytrid pathogenic to amphibians. - Mycologia 91: 219-227.

McCallum, H. et al. 2001. How should pathogen transmission be modelled? - Trends Ecol. Evol. 16: 295-300.

Moss, A. S. et al. 2008. Chemotaxis of the amphibian pathogen Batrachochytrium dendrobatidis and its response to a variety of attractants. - Mycologia 100: 1-5.

Nichols, D. et al. 2001. Experimental transmission of cutaneous chytridiomycosis in dendrobatid frogs. - J. Wildl. Dis. 37: $1-11$.

Piotrowski, J. S. et al. 2004. Physiology of Batrachochytrium dendrobatidis, a chytrid pathogen of amphibians. - Mycologia 96: $9-15$.

Supplementary material (available online as Appendix oik03462 at <www.oikosjournal.org/appendix/oik-03462>). Appendix 1.
Rachowicz, L. J. and Vredenburg, V. T. 2004. Transmission of Batrachochytrium dendrobatidis within and between amphibian life stages. - Dis. Aquat. Organisms 61: 75-83.

Rachowicz, L. J. and Briggs, C. J. 2007. Quantifying the disease transmission function: effects of density on Batrachochytrium dendrobatidis transmission in the mountain yellow-legged frog Rana muscosa. - J. Anim. Ecol. 76: 711-721.

Rachowicz, L. J. et al. 2006. Emerging infectious disease as a proximate cause of amphibian mass mortality. - Ecology 87: $1671-1683$.

Rowley, J. J. and Alford, R. A. 2007. Behaviour of Australian rainforest stream frogs may affect the transmission of chytridiomycosis. - Dis. Aquat. Organisms 77: 1-9.

Schmeller, D. S. et al. 2014. Microscopic aquatic predators strongly affect infection dynamics of a globally emerged pathogen. - Curr. Biol. 24: 176-180.

Tinsley, R. C. et al. 2015. Chytrid fungus infections in laboratory and introduced Xenopus laevis populations: assessing the risks for UK native amphibians. - Biol. Conserv. 184: 380-388.

Tobler, U. and Schmidt, B. R. 2010. Within- and among-population variation in chytridiomycosis-induced mortality in the toad Alytes obstetricans. - PloS ONE 5: e10927.

Tobler, U. et al. 2012. Populations of a susceptible amphibian species can grow despite the presence of a pathogenic chytrid fungus. - PloS ONE 7: e34667.

Venesky, M. D. et al. 2011. Can differences in host behavior drive patterns of disease prevalence in tadpoles? - PloS ONE 6: e24991.

Voyles, J. et al. 2012. Temperature alters reproductive life history patterns in Batrachochytrium dendrobatidis, a lethal pathogen associated with the global loss of amphibians. - Ecol. Evol. 2: 2241-2249.

Walker, S. F. et al. 2007. Environmental detection of Batrachochytrium dendrobatidis in a temperate climate. - Dis. Aquat. Organisms 77: 105-112.

Walker, S. F. et al. 2010. Factors driving pathogenicity vs. prevalence of amphibian panzootic chytridiomycosis in Iberia. - Ecol. Lett. 13: 372-382.

Weldon, C. et al. 2004. Origin of amphibian chytrid fungus. - Emerging Infectious Dis. 10: 2100-2105.

Woodhams, D. C. et al. 2011. Mitigating amphibian disease: strategies to maintain wild populations and control chytridiomycosis. - Front. Zool. 8: 8.

Woodhams, D. C. et al. 2014. Interacting symbionts and immunity in the amphibian skin mucosome predict disease risk and probiotic effectiveness. - PloS ONE 9: e96375. 